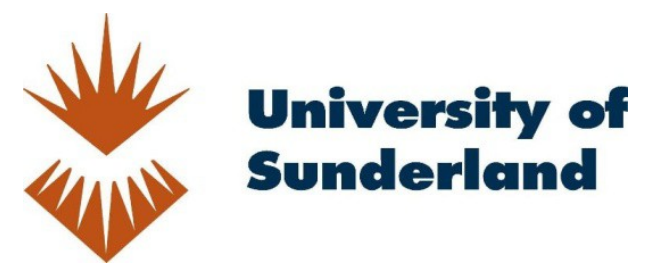

Viti, L., Bianchi, V., Carey, T., Li, L., Linfield, E. H., Davies, A. G., Tredicucci, A., Yoon, D., Karagiannidis, Panagiotis, Lombardi, L., Tomarchio, F., Ferrari, A. C., Torrisi, F. and Vitiello, M. S. (2018) Graphene Saturable Absorbers at Terahertz Frequency from Liquid Phase Exfoliation of Graphite. Conference on Lasers and Electro-Optics, OSA Technical Digest (online) (Optical Society of America, 2018). STu4D.6.

Downloaded from: http://sure.sunderland.ac.uk/id/eprint/9963/ 
Please refer to the usage guidelines at http://sure.sunderland.ac.uk/policies.html or alternatively contact sure@sunderland.ac.uk. 


\title{
Graphene Saturable Absorbers at Terahertz Frequency from Liquid Phase Exfoliation of Graphite
}

\author{
Viti L., ${ }^{1}$ Bianchi V., ${ }^{1}$ Carey T., ${ }^{2}$ Li L., ${ }^{3}$ Linfield E. H., ${ }^{3}$ Davies A. G.,${ }^{3}$ Tredicucci A., ${ }^{4}$ Yoon D., ${ }^{2}$ Karagiannidis \\ P. G., ${ }^{2}$ Lombardi L., ${ }^{2}$ Tomarchio F., ${ }^{2}$ Ferrari A. C., ${ }^{2}$ Torrisi F., ${ }^{2}$ Vitiello M. S. ${ }^{1}$ \\ ${ }^{I}$ NEST, CNR-Istituto Nanoscienze and Scuola Normale Superiore, Piazza San Silvestro 12, Pisa I-56127, Italy. \\ ${ }^{2}$ Cambridge Graphene Centre, University of Cambridge, Cambridge CB3 OFA, UK. \\ ${ }^{3}$ School of Electronic and Electrical Engineering, University of Leeds, Leeds LS2 9JT, UK. \\ ${ }^{4}$ Dipartimento di Fisica, Università di Pisa, Largo Pontecorvo 3, 56127 Pisa, Italy. \\ e-mail address: leonardo.viti@nano.cnr.it
}

\begin{abstract}
We report on the development of terahertz (THz) saturable-absorbers exploiting printable graphene inks. The achieved $80 \%$ transparency modulation at $3.5 \mathrm{THz}$ makes these devices potential candidates as passive components for $\mathrm{THz}$ solid-state lasers.

OCIS codes: (140.5965) Semiconductor lasers, quantum cascade; (250.4390) nonlinear optics, integrated optics; (320.7110)

Ultrafast nonlinear optics.
\end{abstract}

\section{Introduction}

Saturable absorbers (SA) operating in transmission or in refection (saturable absorber mirrors) are routinely used to passively mode-lock lasers, enabling a train of short pulses to be derived from continuous wave operation. The formation of the pulse relies on the non-constant dependence of the SA transmittance with respect to the impinging power: pulses of lower intensity are absorbed more efficiently than those having larger intensities, i.e. the absorber is saturated.

Semiconductor materials can absorb over a broad range of wavelengths (from the visible to the mid-infrared), providing an ideal solution for conceiving SAs. Furthermore, a proper control of the absorption recovery time and saturation fluence can be obtained by altering the growth parameters of the core material and/or the device geometry. However, due to the limited tuning range/bandwidth in reflection and the inherent free-carrier absorption losses in transmission, they are poorly suitable for applications in the $\mathrm{THz}$ frequency range. Devising new technological solutions for the realization of efficient THz SAs can open intriguing frontiers for the development of passively mode-locked THz micro-sources, like, for example, quantum cascade lasers (QCLs), the most powerful and compact sources of THz light [1]. Progress in the field is limited by the absence of suitable materials. In this context graphene appears as an ideal candidate, thanks to the ultrafast carrier dynamics, the frequency independent absorption, the possibility to alter its electrical properties through electrical gating and the fact that it does not need any band gap engineering or chirality/diameter control to optimize or preserve the device performance. Furthermore graphene is flexible and compatible with a broad range of substrate materials.

Graphene-based SAs have been demonstrated in the near infrared, visible and microwave ranges. Saturable absorption, mediated by Pauli blocking effect, has been more recently proven at $\mathrm{THz}$ frequencies [2] by employing turbostratic multilayer graphene grown via chemical vapor deposition (CVD) on the carbon-face of silicon carbide. The quite low absorption modulation (10\%), too low to alter the intra-cavity field of existing THz QCLs and the lack of flexibility for intracavity embedding, practicallymakes this material useless for the mentioned purpose.

Here we demonstrate THz saturable absorption in graphene films produced by either vacuum filtration or ink-jet printing on silicon. Through the combination of open-aperture z-scan experiments, transport analysis of field effect transistors embedding the devised inks and Fourier transform infrared (FTIR) spectroscopy, we demonstrate 80\% transparency modulation. The ultrafast $(\sim 100 \mathrm{fs})$ carrier-carrier scattering mechanism, which dominates intraband transitions, together with the intrinsic ease of fabrication, handling and deposition, paves the way for the integration of graphene with existing sources to realize ultrafast, mode-locked lasers, and passive ultrafast components across the $\mathrm{THz}$ frequency range.

\section{Experiments and results}

We formulate a low-boiling point graphene-ink, and we produced graphene SAs by either vacuum filtration or inkjet printing on silicon. Two samples are here presented. The first graphene saturable absorber (GSA) is prepared with an ethanol-based ink (GEth), which is inkjet printed on an intrinsic silicon substrate, capped by $300 \mathrm{~nm}$ of $\mathrm{SiO}_{2}$. Figure 1a presents a high-resolution transmission electron microscopy (HRTEM) image of single and few- 
layer graphene flakes from the ethanol-based ink. The second GSA is prepared with water-based ink $(\mathrm{GW})$, which is water filtered on a $\mathrm{Si} / \mathrm{SiO}_{2}$ substrate. Figure $1 \mathrm{~b}$ shows the HRTEM image graphene flakes from the water-based ink. The average lateral size and thickness of the GW and GEth flakes were estimated by atomic force microscopy (AFM). Raman spectroscopy was used to monitor the quality of the flakes at each step of the GSA preparation process for both inks; specifically the analysis of the Raman peaks allowed to discriminate between disorder localized at the edges or in the bulk of the samples and to extract Fermi energy and, correspondingly, the doping level. Fourier transform infrared spectroscopy was then used to determine the linear absorption of the GSAs in the THz, MIR and near-IR frequency ranges and to evaluate the carrier scattering dynamics of the GSAs.

To investigate the THz-induced non-linear absorption properties of the devised GSA we employed an openaperture $z$-scan technique. The $3.5 \mathrm{THz}$-frequency radiation generated by a high power single-plasmon QCL was focused on the samples surface along the direction orthogonal to the substrate. The samples were then translated along the optical axis ( $z$ direction), while measuring the transmitted $\mathrm{THz}$ power. The scan along the $z$ direction corresponds to exposing the samples to different $\mathrm{THz}$ intensities, inversely proportional to the beam spot area impinging on the surface. With this technique, we retrieved transmittance profiles demonstrating the saturation of absorption for high $\mathrm{THz}$ intensities, i.e. in proximity of the focal point. Figure 1c presents the normalized transmittance profile obtained for the water-based GSA, showing a transparency modulation of the $80 \%$ [3].
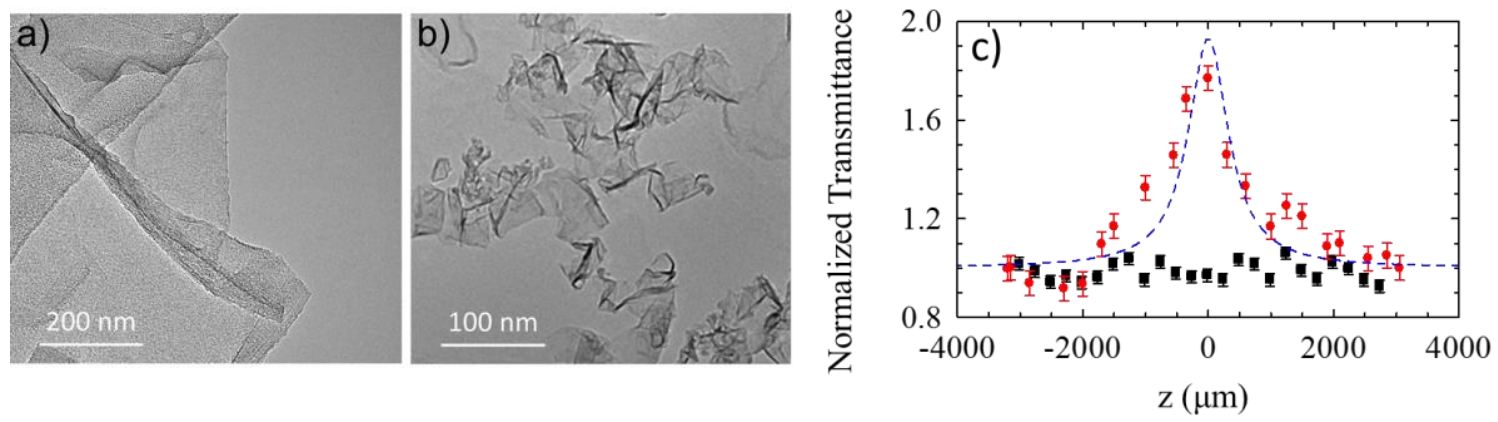

Fig.1. (a) High-resolution transmission electron micrograph (HRTEM) of graphene flakes from ethanol based ink. (b) HRTEM image of graphene flakes from water based ink. (c) Z-scan normalized transmittance profile of the water based graphene saturable absorber (red dots), compared with z-scan normalized transmittance traces of the $\mathrm{Si}_{/} / \mathrm{SiO}_{2}$ substrate (black squares). The results are fitted with a two-level saturable absorber model, represented by the dashed line.

\section{Conclusions}

The demonstrated $80 \%$ transparency modulation combined with the flexibility offered by graphene films and inks and the fast absorption dynamics prove the potential of ink-based GSAs for the engineering of new classes of photonic devices in the $\mathrm{THz}$ frequency range with specifically designed device functionalities. The latter include ultrafast $\mathrm{THz}$ modulators, filters and mirrors, or $\mathrm{THz}$ passive components that can be easily integrated with existing laser systems to drive them in the mode-locking regime. This can open novel frontiers for appealing application fields as time-of-flight tomography, coherent manipulation of quantum systems, time-resolved spectroscopy of gases, complex molecules and cold samples or ultra-high speed communication.

\section{Acknowledgements}

We acknowledge funding from ERC grants SPRINT, Hetero2D, HiGRAPHINK, the EU Graphene Flagship, and EPSRC grants EP/K01711X/1, EP/K017144/1, EP/L016087/1, EP/N010345/1, EP/P02534X/1

\section{References}

[1] M. S. Vitiello, G. Scalari, B. Williams and P. De Natale, “Quantum cascade lasers: 20 years of challenges,” Opt. Express 23, 5167-5182 (2015).

[2] F. Bianco, V. Miseikis, D. Convertino, J.H. Xu, F. Castellano,H. E. Beere, D. A. Ritchie, M. S. Vitiello, A.Tredicucci, and C. Coletti, "THz saturable absorption in turbostratic multilayer graphene on silicon carbide", Optics Express 23, 11632 (2015).

[3] V. Bianchi, T. Carey, L. Viti, L. Li, E. H. Linfield, A. G. Davies, A. Tredicucci, D. Yoon, P. G. Karagiannidis, L. Lombardi, F. Tomarchio, A. C. Ferrari, F. Torrisi, M. S. Vitiello, "Terahertz saturable absorbers from liquid phase exfoliation of graphite”, Nature Comm. 8, 15763 (2017). 